\title{
Cluster observations on the thin current sheet in the magnetotail
}

\author{
C. L. Cai ${ }^{1,2}$, I. Dandouras ${ }^{1}$, H. Rème ${ }^{1}$, J. B. Cao ${ }^{2}$, G. C. Zhou ${ }^{2}$, and G. K. Parks ${ }^{3}$ \\ ${ }^{1}$ Centre d'Etude Spatiale des Rayonnements, Centre National de la Recherche Scientifique/Université Paul Sabatier, Toulouse \\ 31028 , France \\ ${ }^{2}$ State Key Laboratory of Space Weather, Center for Space Science and Applied Research, Beijing 100080, China \\ ${ }^{3}$ Space Sciences Laboratory, University of California, Berkeley, CA, 94720, USA
}

Received: 8 May 2007 - Revised: 19 February 2008 - Accepted: 3 March 2008 - Published: 13 May 2008

\begin{abstract}
A magnetotail event on 15 September 2001 is analyzed, during which the Cluster spacecraft observed both embedding and bifurcation of the Thin Current Sheet (TCS). It is indicated that the ion anisotropy and nongyrotropy are responsible for those new equilibrium features that represent deviations from the conventional Harris model. Measurements show that an embedded proton TCS manifests a pressure anisotropy with $p_{\|}>p_{\perp}$, simultaneously associated with a density embedding; while a bifurcated oxygen ion TCS exhibits a pressure anisotropy mainly with $p_{\|}<p_{\perp}$ and nongrotropy, except at the edges where $p_{\|}>p_{\perp}$ is the necessary marginal firehose stability condition. The local flapping motion of the TCS was observed, and some particular features such as the solitary wave-like behavior and the kink motion in the plane perpendicular to the Sun-Earth direction were revealed. The present investigation intimates the complexity of equilibria of the magnetotail current sheet and declares the crucial role played by the ion kinetics in the dynamics of TCSs.
\end{abstract}

Keywords. Magnetospheric physics (Magnetotail; Plasma sheet) - Space plasma physics (Nonlinear phenomena)

\section{Introduction}

The understanding of the dynamics of the magnetotail current sheet is important to many space phenomena. The conventional current sheet model is based on the Harris equilibrium, which has the well-known tanh-type magnetic field profile and the bell-shaped density and current profile, that is, current and plasma densities have a simple profile with a single peak in the center of the sheet where the magnetic field has a minimum (Harris, 1962). The Harris equilibrium is in good agreement with the observations of the thick, quiet-

Correspondence to: C. L. Cai

(clcai@cssar.ac.cn) time current sheet (Fairfield, 1979; Thompson et al., 2005). However, TCSs, whose thicknesses are on the order of the ion gyroradius or inertial length, were reported recently by mumerous studies (Sergeev et al., 1993; Hoshino et al., 1996; Runov et al., 2003a, 2005; Sergeev et al., 2003). Behaviors of TCS deviated significantly from Harris's equilibrium. Observations revealed that in some instances, TCSs have a manifestation of embedding, which means that TCSs with rather large current densities are embedded into a much thicker current sheet, and the thicker current sheet is usually in accord with a Harris equilibrium. In some other cases, TCSs have a bifurcated structure, that is, TCSs commonly have a doublepeak, sometime multi-peak, current profile, and the current density has a minimum at the center of the sheet. TCSs are frequently occurring phenomena in the magnetotail and hence raise a question about the interpretation of their formation and evolution (Asano et al., 2005).

Many theoretical models have attempted to describe the TCS structures and dynamics. In one category, an isotropic pressure is assumed. These models either choose the particle distributions to be a function of two invariants of motion, namely the total particle energy and the component of the canonical momentum along the current direction, as in the Harris model but in their generalized non-Maxwellian forms, or adopt equivalently the Grad-Shafranov equation, in which the current density is a function of the electromagnetic field vector potential (Schindler et al., 2002; Mottez, 2003; Birn et al., 2004a; Genot et al., 2005; Camporeale et al., 2005). Therefore, it implies that in all of them, the pressure is isotropic, and the equilibrium is maintained by a balance between the field line tension and the plasma pressure gradient. In the other category, an anisotropic pressure is assumed, hence the equilibrium is achieved for a one-dimension current sheet only if the magnetic tension is balanced by the tension due to the ansiotropic pressure, namely the finite ion inertia (Eastwood, 1972). It was pointed out that in this case the current is confined to a thin layer, in which the particle

Published by Copernicus Publications on behalf of the European Geosciences Union. 

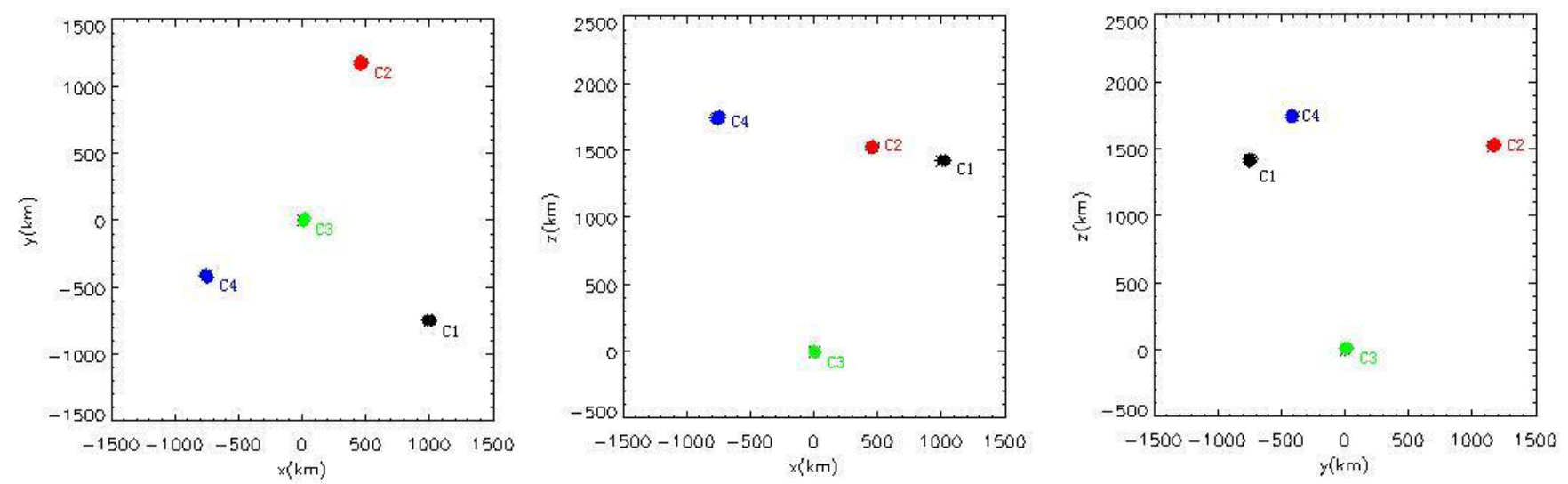

Fig. 1. The configuration of Cluster tetrahedron in the time interval 04:40-05:10 UT on 15 September 2001.

motion may strongly differ from Larmor rotation, i.e. the particle dynamics does not obey the conventional guiding center theory, when its gyroradius becomes comparable to either the current sheet thickness or the curvature radius of the magnetic field. Behavior of these nonadiabatic ions is known as the Speiser motion (Speiser, 1965). Nonadiabatic ions play an important role in the current sheet dynamics, and their kinetics in the TCS have been investigated intensely (Delcourt et al., 2004, 2006; Birn et al., 2004b). Moreover, when the current sheet is thin enough to obey some appropriate conditions, an integral of motion, the so-called quasi-adiabatic sheet invariant can be introduced (Sonnerup, 1971). Recently, a stationary state Vlasov theory involving the quasiadiabatic sheet invariant was proposed to describe the TCS (Sitnov et al., 2000, 2003, 2006). Choosing the distribution of counterstreaming beams in the outer part of the sheet, the numerical results show that a bifurcated current sheet appears in the case of ion anisotropy with $T_{\perp}>T_{\|}$; while in the opposite case a single-peak current sheet embedded in a thicker Harris current sheet appears.

In addition, TCS is frequently found in association with rapid, large-amplitude magnetic variations, namely a flapping motion indicates rapid crossings through an up-down oscillating current sheet (Sergeev et al., 2003; Runov et al., 2003a, 2005). The flapping motion was identified to be a kink-like wave propagating in the current sheet direction. Statistical investigations revealed its particular propagating features. The propagating speeds are in the range of several tens of $\mathrm{km} / \mathrm{s}$ up to $200 \mathrm{~km} / \mathrm{s}$. The propagating direction is flankward, that is, dawnward in the dawn sector and duskward in the dusk sector (Sergeev et al., 2004; Zhang et al., 2005). As pointed out, the wave properties do not match any local excitation mechanism previously discussed so far in the literature (Zhu et al., 1996; Daughton, 1999, 2002, 2003; Lapenta et al., 2002). It raises another question to understand the dynamics of TCSs.

Here, TCS dynamics is reported from observations during the Cluster spacecraft crossing of the magnetotail on 15
September 2001. First, an overview of the entire event, including the spacecraft configuration and characteristic parameters, is presented. Then various manifestations of TCSs in association with their ion anisotropy and nongyrotropy, as well as their flapping motion are analyzed. Finally, a discussion and summary are given.

\section{Observations}

\subsection{Overview}

In the time interval 00:00-06:00 UT on 15 September 2001, the IMF $B_{z}$ was mainly northward, turned southward shortly at about 03:20 UT and during 04:30-05:00 UT, and again shortly southward at about 05:30 UT (The solar wind parameters come from the ACE data and aren't shown here). The solar wind velocity had no apparent change. A substorm was observed during 00:00-00:50 UT, which the AL index was up to $-700 \mathrm{nT}$ and then decreased to zero. The geomagnetic activity started again at 03:40 UT. During the interval of 03:30-06:30 UT, several auroral activations were registered by two geostationary satellites, GOES 8 and GOES 10 (Voronkov et al., 2006).

The configuration of Cluster tetrahedron in the period 04:40-05:10 UT is shown in Fig. 1. The characteristic scale of the tetrahedron is $1700 \mathrm{~km}$. It is worth to noticing that in the $\mathrm{x}-\mathrm{z}$ plane, $\mathrm{C} 3$ is southmost and the other three satellites have small distances among them in the z-direction; all four satellites are in the dusk sector and in the y-z plane $\mathrm{C} 2$ is outermost. Characteristic parameters of the background plasma and fields in this event are $T_{H}=2 \mathrm{keV}, T_{O}=20 \mathrm{keV}, B_{0}=30 \mathrm{nT}$ and $n_{i}=0.5 \mathrm{~cm}^{-3}$, where $T_{H}$ and $T_{O}$ are the temperatures of proton and oxygen ions, respectively, $B_{0}$ is the magnetic field in the periphery of the plasma sheet and $n_{i}$ is the total ion density. Thus, we have approximately $\rho_{H}=220 \mathrm{~km}$, $\rho_{O}=2500 \mathrm{~km}$ and $c / \omega_{p i}=320 \mathrm{~km}$, where $\rho_{H}$ and $\rho_{O}$ are the gyroradius of proton and oxygen ions, respectively, and 
$15 / \operatorname{sep} / 2001$

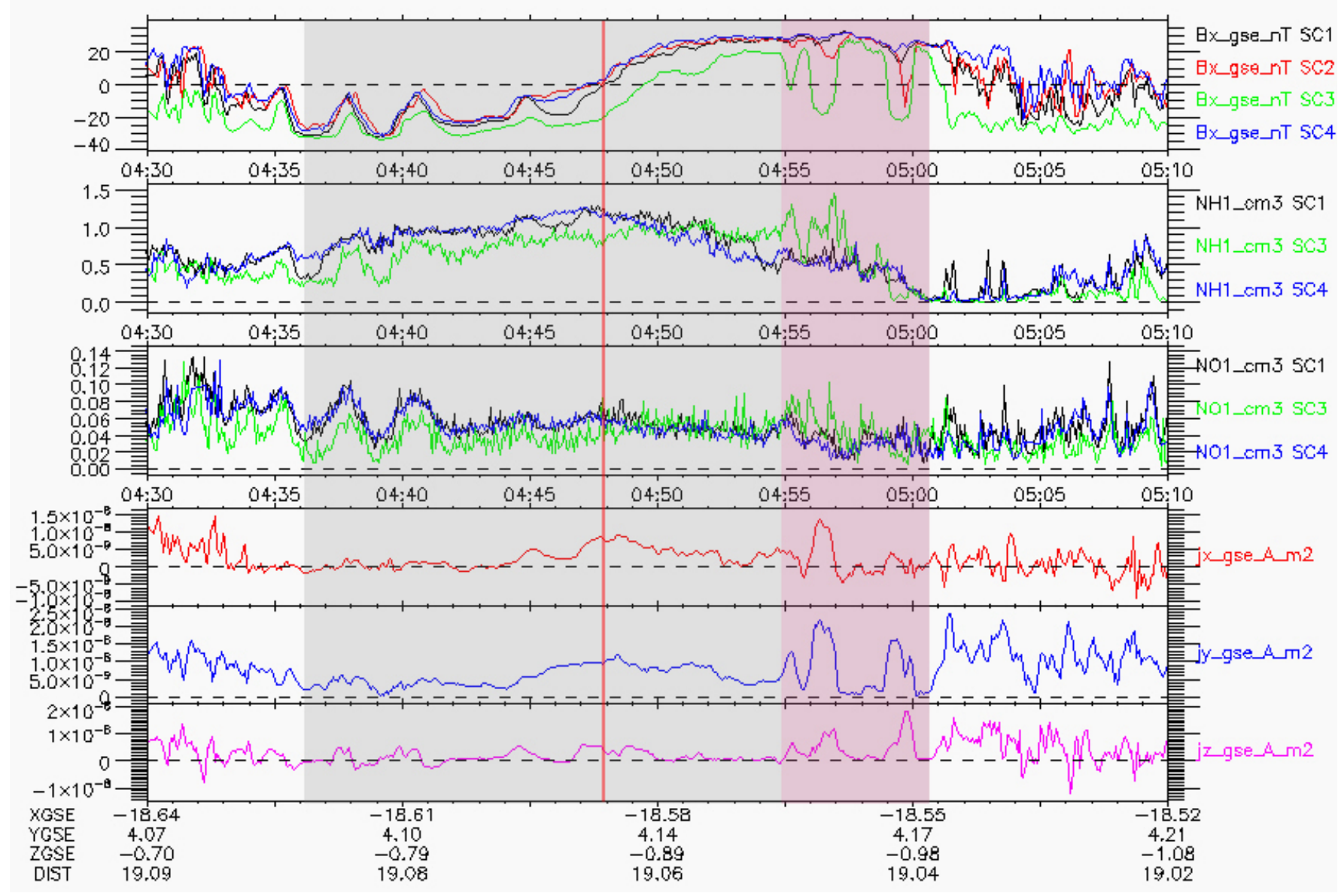

Fig. 2. Overview of the Harris-like current sheet. (a) $B_{x}$ for four satellites. (b) and (c) are the proton and oxygen ion densities, respectively. (d-f) are the current density components.

$c / \omega_{p i}$ is the ion inertial length. The characteristic scale of the Cluster tetrahedron is comparable to the oxygen ion gyroradius and is almost five times the ion inertial length.

Figure 2 shows the components of magnetic field from the FGM experiment and the proton and oxygen ion densities from the CIS-CODIF instrument (Balogh et al., 2001; Rème et al., 2001). As represented by the whole shading region in the interval 04:36-05:01 UT in Fig. 2, all four satellites recorded a bell-shaped plasma density and current profiles, whose peaks are corresponding to the minimum of the magnetic field. Hence it is indicated that a Harris-like current sheet was encountered. C1, C2 and C4 crossed almost simultaneously the central line at 04:48 UT, as labeled by a vertical line, due to a global flapping motion of the bulk current sheet. A little later C3 crossed the central line at 04:50 UT. However, compared to the Harris-like equilibrium with a larger thickness, in the interior of the thick sheet, as shown by the narrow red shading, distinct manifestations of localized thin current equilibrium are encountered. Approximately in the interval 04:55-04:59 UT, a thin proton dominating the

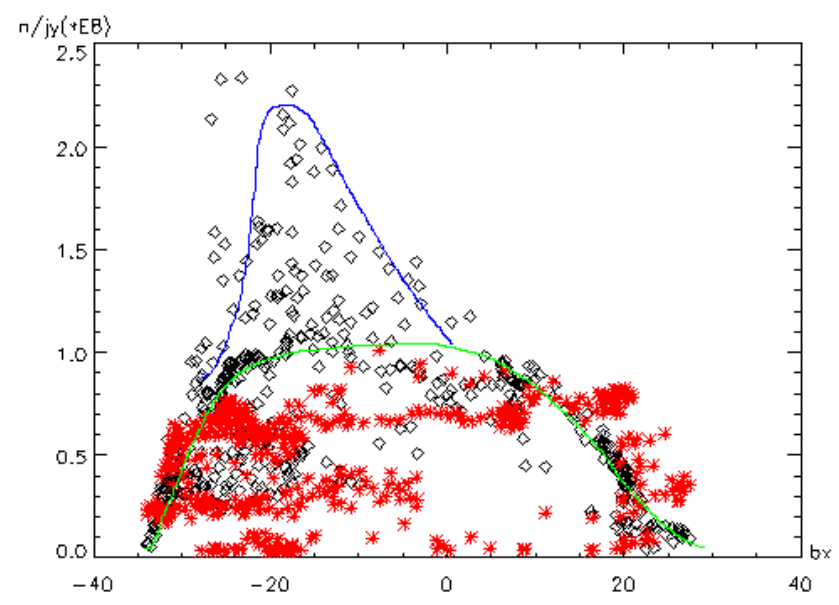

Fig. 3. The scattering plot of the current density together with the proton density versus $B x$. 


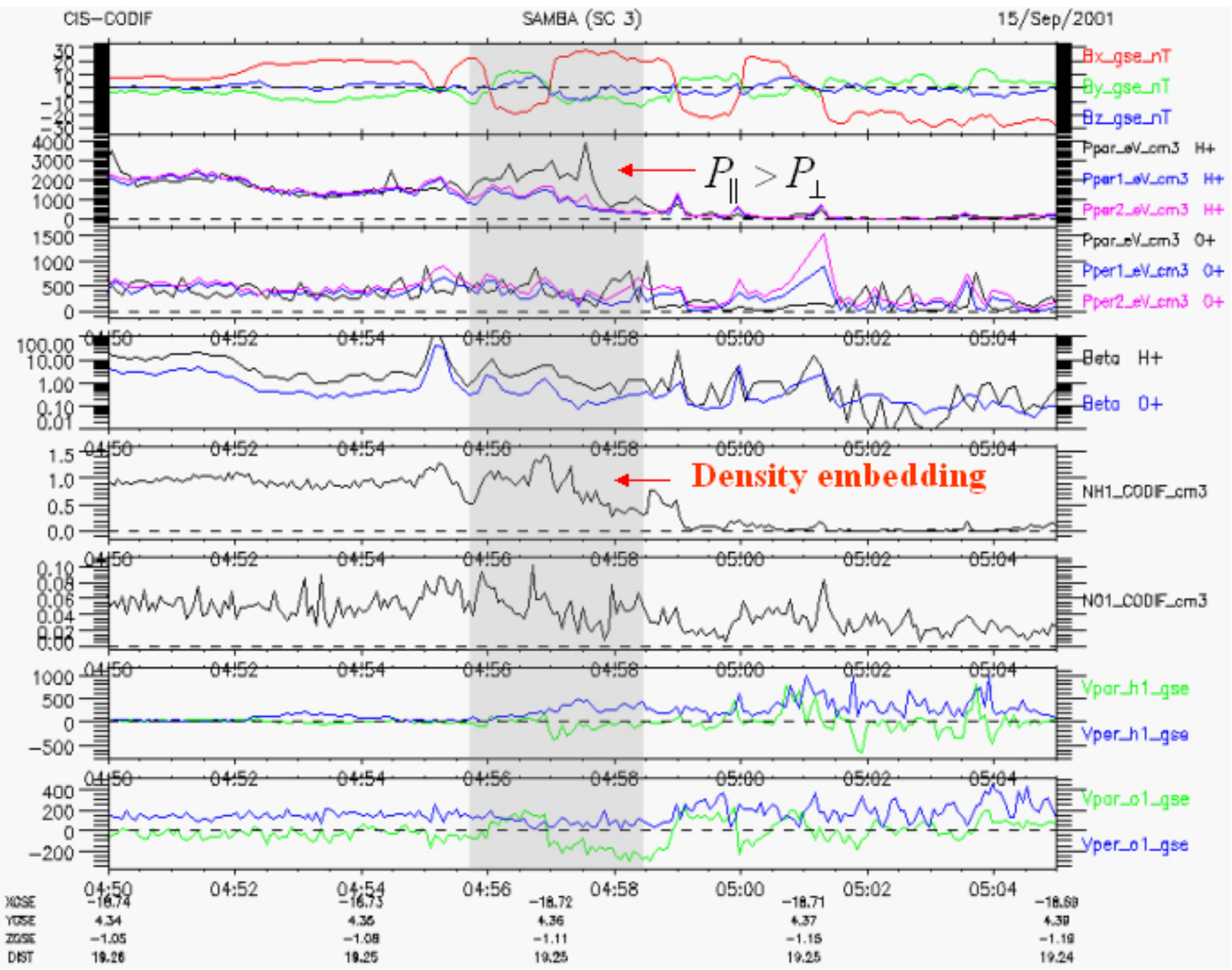

Fig. 4. 3-D momenta of proton and oxygen ions from C3. (a) the components of magnetic field. (b) and (c) are the pressure components. (d) is the beta value. (e) and (f) are the densities. (g) and (h) are bulk velocities.

current sheet which was embedded into the southern part of the thick sheet was observed by $\mathrm{C} 3$. In addition, nearly in the time interval 04:58-05:00 UT, a bifurcated thin oxygen ion current located in the northern part of the thick sheet was observed by $\mathrm{C} 1$ and $\mathrm{C} 4$. Here, it is worth noticing that a plasma depletion layer was subsequently generated inside the plasma sheet. It can be seen that in the second panel in Fig. 2, C3 at 04:59:00 and $\mathrm{C} 1$ and $\mathrm{C} 4$ at about 05:00:20, all of them observed a density decrease nearly to zero till 05:05:20.

\subsection{TCS embedding}

The embedding feature of the proton dominated TCS was revealed in the scattering plot of the y-component of the current density (the total current density profile is similar to its y-component), together with the proton density versus $B_{x}$. As shown in Fig. 3, the red stars represent the proton density, and the black squares denote the current density in the ydirection (it is the average current density inside the satellite tetrahedron and is multiplied by a factor $10^{8}$, the units of current and ion density are $\mathrm{A} / \mathrm{m}^{2}$ and $\mathrm{cm}^{-3}$, respectively). The green curve describes a fitting of the thick Harris-like current sheet, and the blue one depicts a fitting of the thin current sheet. It can be seen that the two current profiles are distinct, where the peak of the green curve is contained by the peak of blue curve. The ion density profile has a width that is roughly equal to that of the wider current profile. Hence the situation here is just the manifestation of the TCS embedding, which means that a thin current sheet with large current density is embedded into the southern part of a wider current sheet.

In the time interval 04:55:40-04:58:30 UT, as shown by the shading in Fig. 4, C3 measured continuously an anisotropic but gyrotropic pressure with $p_{\|}>p_{\perp}$ for protons, while at the rest time it observed an apparent isotropic 


\section{CIS-CODIF SAMBA (SC 3) 15/Sep $/ 2001$}
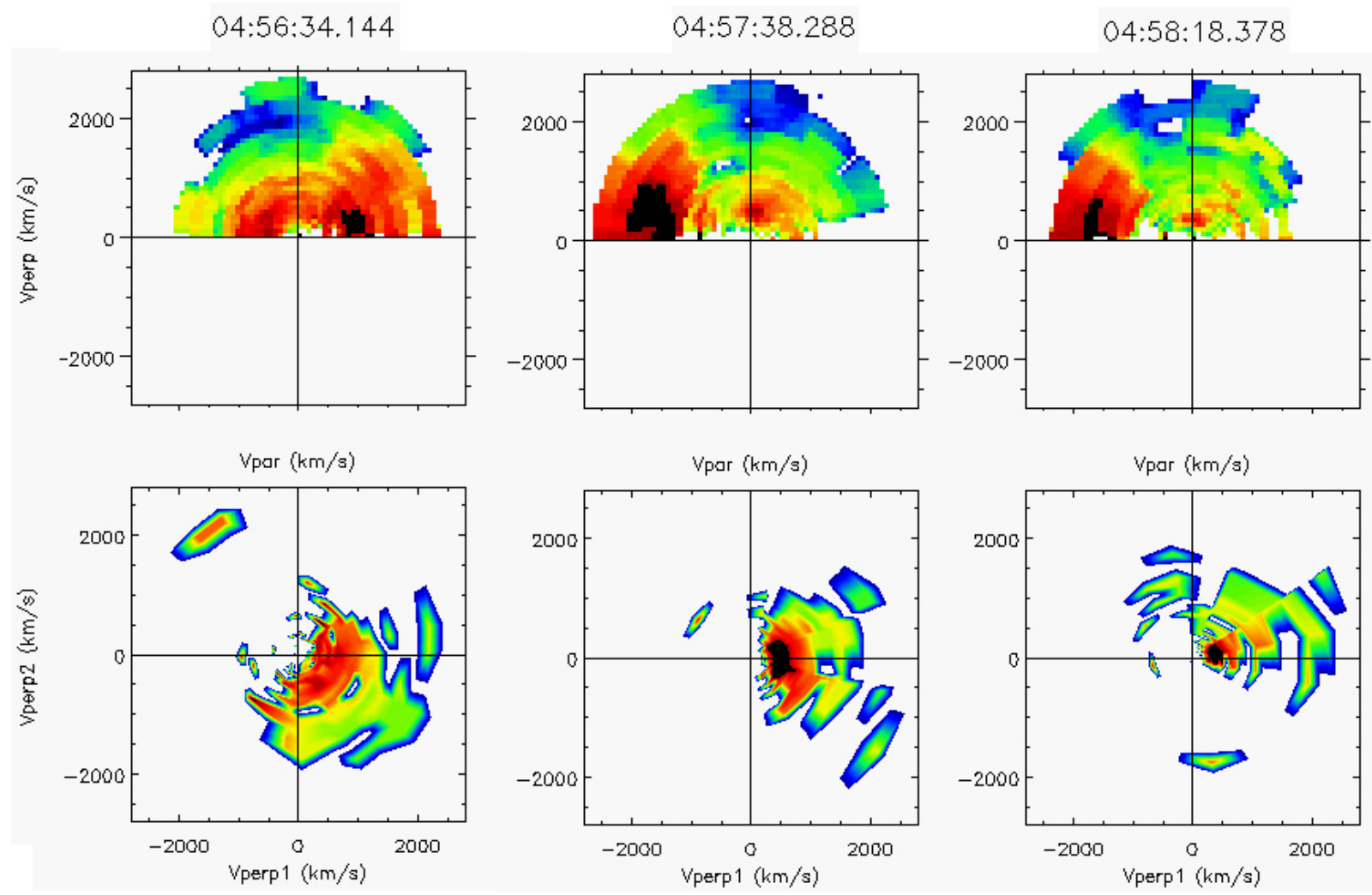

Fig. 5. Distribution functions of protons in the $V_{\|}-V_{\perp}$ coordinates from $\mathrm{C} 3$ in the embedded TCS.

pressure. Simultaneously, the oxygen ion pressure was also nearly isotropic with negligible fluctuations. Figure 5 shows selected proton distribution functions at the beginning, the middle and the end of this period. All of them exhibited an apparent field-aligned beam distribution. It is worth noticing that although a strong pressure anisotropy existed here, it still didn't achieve $p_{\|} / p_{\perp}>1+2 / \beta_{\perp}$, which is the critical condition for exciting the firehose instability (Hasegawa, 1975). As pointed out by comparing the current distribution in the outer and in the center of the sheet, the current carried by a field-aligned beam satisfying the marginal firehose stability condition has a strong tendency to become thin, till a nonadiabatic current layer is formed (Eastwood, 1972; Cowley, 1978). Namely, when there is pressure anisotropy caused by field-aligned beams with $p_{\|}>p_{\perp}$, the current sheet should become an embedded nonadiabatic thin layer, in which an equilibrium is achieved when the magnetic tension is balanced by the finite inertia of ions with meandering Speiser motion. Here it is also worth noticing that the proton pressure is just only anisotropic and that no apparent nongyrotropy was detected. This seems to be contradictory since the ion motion should be nongyrotropic inside the TCS (Sitnov et al., 2006). We infer that ions are strongly nongyrotropic only in the center of the TCS but slightly nongyrotropic in the more outer part of the TCS. In the present situation, the satellite didn't stay close to the center of the TCS except for several rapid crossings of the central line, due to the flapping motion that will be shown in the following. It can also be seen from the fact that in panel (d) in Fig. 4, in this period, $\beta_{H}$ was moderate and no more than 1 , while it was over 10 when the satellite was closer to the central line. This can explain why no apparent pressure nongyrotropy was observed. Apart from the current embedding, a plasma density embedding was also recorded. A proton density embedding is exhibited in panel (e) in Fig. 4, while the oxygen ion density hasn't this manifestation. Although the concrete mechanism of density embedding isn't clear, there is an apparent correlation between the density embedding and the pressure anisotropy with $p_{\|}>p_{\perp}$.

\subsection{TCS bifurcation}

As to be expected, the effects of spatial and temporal nonadiabaticity and nongyrotropy are much more pronounced for the heavy ions than for the lighter ions and the electrons (Birn et al., 2004b). Nearly in the time interval 


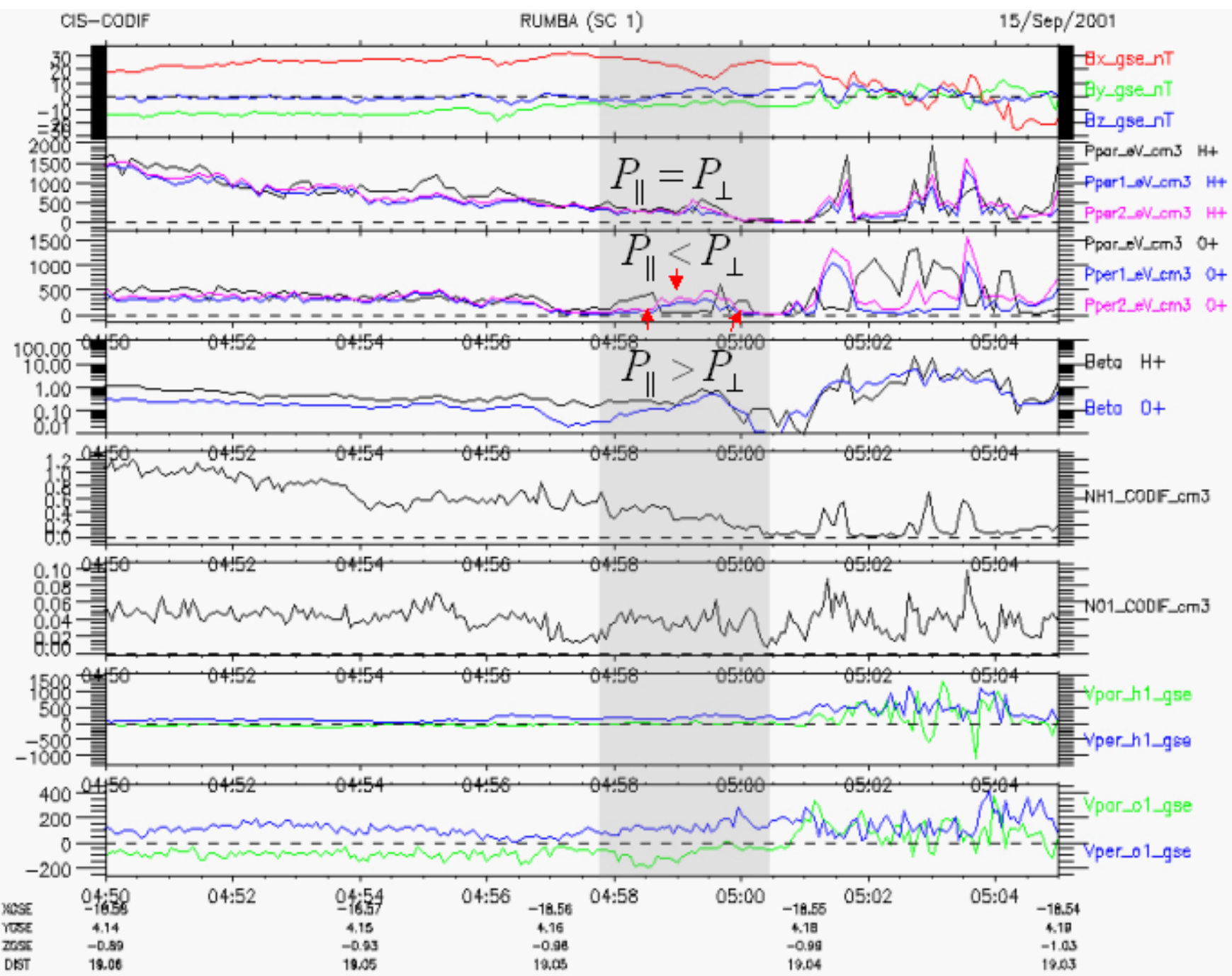

Fig. 6. 3-D momenta of proton and oxygen ions from $\mathrm{C} 1$. (a) the components of magnetic field. (b) and (c) are the pressure components. (d) is the beta value. (e) and (f) are the densities. (g) and (h) are bulk velocities.

04:57:45-05:00:25 UT, both the energy spectrogram and the pitch angle profile (they aren't shown here) reveal that a localized self-consistent current sheet equilibrium of oxygen ions was observed by $\mathrm{C} 1$ and $\mathrm{C} 4$, although in this very thin sheet the current contribution from oxygen ions is minor. We notice that due to the bulk excursion of the thicker Harris-like sheet (it is the up-down motion of the entire plasma sheet and is not the local flapping motion of the TCS observed by $\mathrm{C} 3$ ), the time difference of encounter of the above-mentioned plasma depletion layer by $\mathrm{C} 3$ and $\mathrm{C} 1$ (at 04:59:05 and 05:00:25 UT, respectively), which have a distance of $1400 \mathrm{~km}$ in the z-direction between them, is about $80 \mathrm{~s}$; hence, the shift in velocity of the plasma sheet is about $17 \mathrm{~km} / \mathrm{s}$. The duration of the crossing of the oxygen TCS is about $150 \mathrm{~s}$, thus the estimation of the thickness of the oxygen TCS is about $2500 \mathrm{~km}$. This is approximately one oxygen ion gyroradius and eleven times the proton gyrora- dius. Hence, the localized oxygen ion current equilibrium is an extra-thin current sheet (Sitnov et al., 2006).

In the time interval 04:57:45-05:00:25 UT, as shown by the shading in Fig. 6, C1 measured an oxygen ion pressure anisotropy with $p_{\|}<p_{\perp}$ and nongyrotropy $p_{\perp 1} \neq p_{\perp 2}$ in the center of TCS, however in two edges the pressure is $p_{\|}>p_{\perp}$ and nearly gyrotropic. The proton pressure in this time inter$\mathrm{val}$ is isotropic at a surprising level. As mentioned above, the ion motion is the non-adiabatic, meandering Speiser motion in the TCS. Moreover, when the current sheet is thin enough to obey $L \ll \rho_{0}\left(B_{0} / B_{n}\right)^{2}$ (where $\rho_{0}$ is the particle gyroradius and $B_{0}$ and $B_{n}$ are the magnetic field at the outer part of the current sheet and its normal component in the center of sheet, to be satisfied definitely for the oxygen ion current sheet in the present situation), the motion of transient particles will be multi-crossings of the sheet, that is, a fast bounce motion across the thin sheet. In this case, the particle dynamics 
CIS-CODIF RUMBA (SC 1) 15/Sep/2001
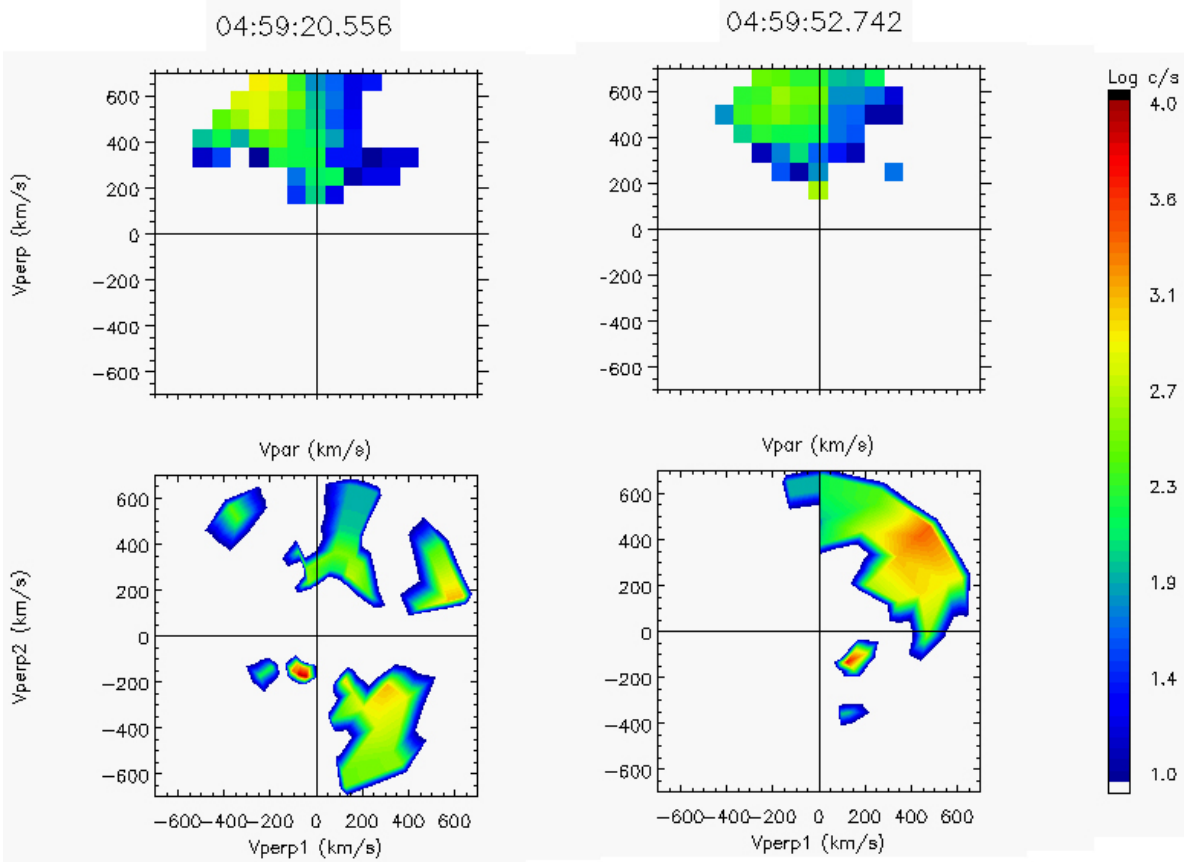

Fig. 7. Distribution functions of oxygen ions in the $V_{\|}-V_{\perp}$ coordinates from $\mathrm{C} 1$ in the bifurcated TCS.

becomes approximately adiabatic or "quasi-adiabatic" along the normal direction of the sheet, and the so-called quasiadiabatic sheet invariant can be introduced (Sonnerup, 1971). As a consequence, the major motion of particles in the center of the TCS will be in the perpendicular direction. Figure 7 shows the oxygen ion distributions selected in the center of the thin sheet. It states the fact that the oxygen ion motion in the perpendicular direction is dominant. Combined with the necessary marginal firehose stability condition at the edge, it is exactly the particular observational feature in the present situation, that is, an oxygen ion pressure anisotropy with $p_{\|}<p_{\perp}$ and nongyrotropy in the center of the TCS, but a gyrotropic pressure anisotropy with $p_{\|}>p_{\perp}$ at edges. Moreover, non-adiabatic particles belonging to different classes with distinct dynamics in the phase space have different current carrying capabilities (Chen et al., 1986; Chen, 1992; Buchner et al., 1989; Burkhart et al., 1991; Zelenyi et al., 2002). The scattering of particle adiabatic invariant will result in the variation of the population of non-adiabatic particle and accordingly the current bifurcation. A detailed analysis on the particle kinetics will be presented elsewhere.

In the time interval 04:58:00-05:00:05 UT, C4 recorded very similar features concerning the oxygen ion TCS as $\mathrm{C} 1$, as shown in Fig. 8. The characteristics of oxygen ion distribution functions in the center of TCS are also like that observed by $\mathrm{C} 1$, as shown in Fig. 9. We notice here that the distance between $\mathrm{C} 1$ and $\mathrm{C} 4$ in the Sun-Earth direction is the largest distance of the Cluster tetrahedron along the field line and that the distance in the normal direction between them is very small. The similarity of measurements between $\mathrm{C} 1$ and C4 imply that the variance in the Sun-Earth direction is negligible, that is, the TCS can be described qualitatively by a one-dimension model. Also, absolute values of the plasma pressures recorded by $\mathrm{C} 1$ and $\mathrm{C} 4$ are nearly equal. This means that there is no pressure gradient in the field line direction. However, the pressure gradient is the necessary condition for TCS models with the isotropic pressure, in the case that the normal component of magnetic field is nonzero.

\subsection{Flapping motion}

TCS is frequently found in association with rapid largeamplitude magnetic variations, namely a flapping motion indicating rapid crossings through the up-down oscillating current sheet. During the period of 04:55-05:02 UT, four rapid large-amplitude $B_{x}$ variations were observed by $\mathrm{C} 3$, as labeled by the shadings in Fig. 10. Their peaks were at 04:55:10, 04:56:30, 04:59:35 and 05:01:30 UT, respectively. The $B_{x}$ variations were also apparently recorded, but weaker, in phase by $\mathrm{C} 2$; while $\mathrm{C} 1$ measured in phase the perceptible $B_{x}$ variations only at the third and fourth oscillations, and $\mathrm{C} 4$ nearly recorded nothing except a variation with a negligible amplitude at the third oscillation. These magnetic variations are the manifestation of kink-like wave propagating in the dawn-duck direction (Sergeev et al., 2003; Runov et al., 


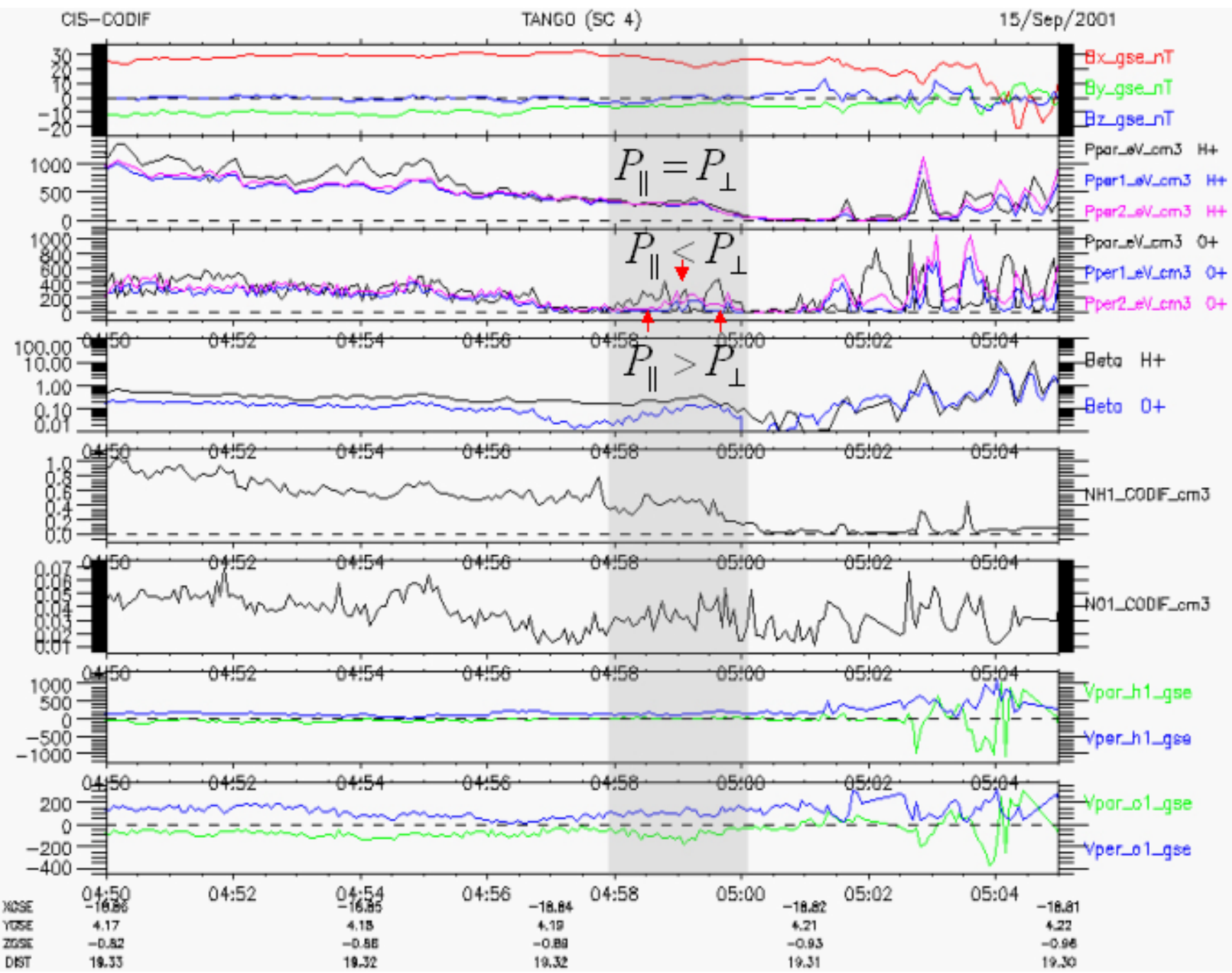

Fig. 8. 3-D momenta of proton and oxygen ions from C4. (a) the components of magnetic field. (b) and (c) are the pressure components. (d) is the beta value. (e) and (f) are the densities. (g) and (h) are bulk velocities.

2003a, 2005). Utilizing the time difference of the peak of magnetic variations between $\mathrm{C} 3$ and $\mathrm{C} 2$, we can estimate its phase speed to be $120 \mathrm{~km} / \mathrm{s}, 50 \mathrm{~km} / \mathrm{s}, 30 \mathrm{~km} / \mathrm{s}$ and $40 \mathrm{~km} / \mathrm{s}$ corresponding to four oscillations, respectively. The periods of the four oscillations are $70 \mathrm{~s}, 190 \mathrm{~s}$ and $125 \mathrm{~s}$, respectively: one value is missing. The wavelength is approximately $0.8 \sim 1.5 R_{E}$. The propagating speed estimated here is in accord with the previous statistical investigations, that is, in the range of several tens $\mathrm{km} / \mathrm{s}$ up to $200 \mathrm{~km} / \mathrm{s}$ (Sergeev et al., 2004; Zhang et al., 2005). Moreover, the present event reveals explicitly the solitary wave feature of the flapping motion. For the first and fourth oscillations, the flapping amplitude is smaller than that of the second and third oscillations. Recalling that the period and phase speed have also a similar feature, it is apparent that the behavior of flapping motion resembles a solitary wave modulated both on its amplitude and frequency.
Due to the localized large-amplitude kink motion, the current would have a circular flow in the $y-z$ plane. This point can also be seen in the simulation work (Sitnov et al., 2006). Via the current density and its components shown from panels (d) to (g) in Fig. 10, circular flows corresponding to the second and third oscillations can be discovered. As labeled by a solid vertical line, at each circular flow, the peak of $j_{z}$ is not corresponding to the peak of $j_{y}$ and $j_{x}$, but corresponding to the edge of them. It is just about the manifestation of the current circular flow. At that time, the current sheet is tilted. As shown on panels (b) and (c), for the second and third oscillations, $\mathrm{C} 3$ and $\mathrm{C} 2$ recorded large-amplitude $B_{y}$ or $B_{z}$ variations, reflecting the localized current sheet inclination. 


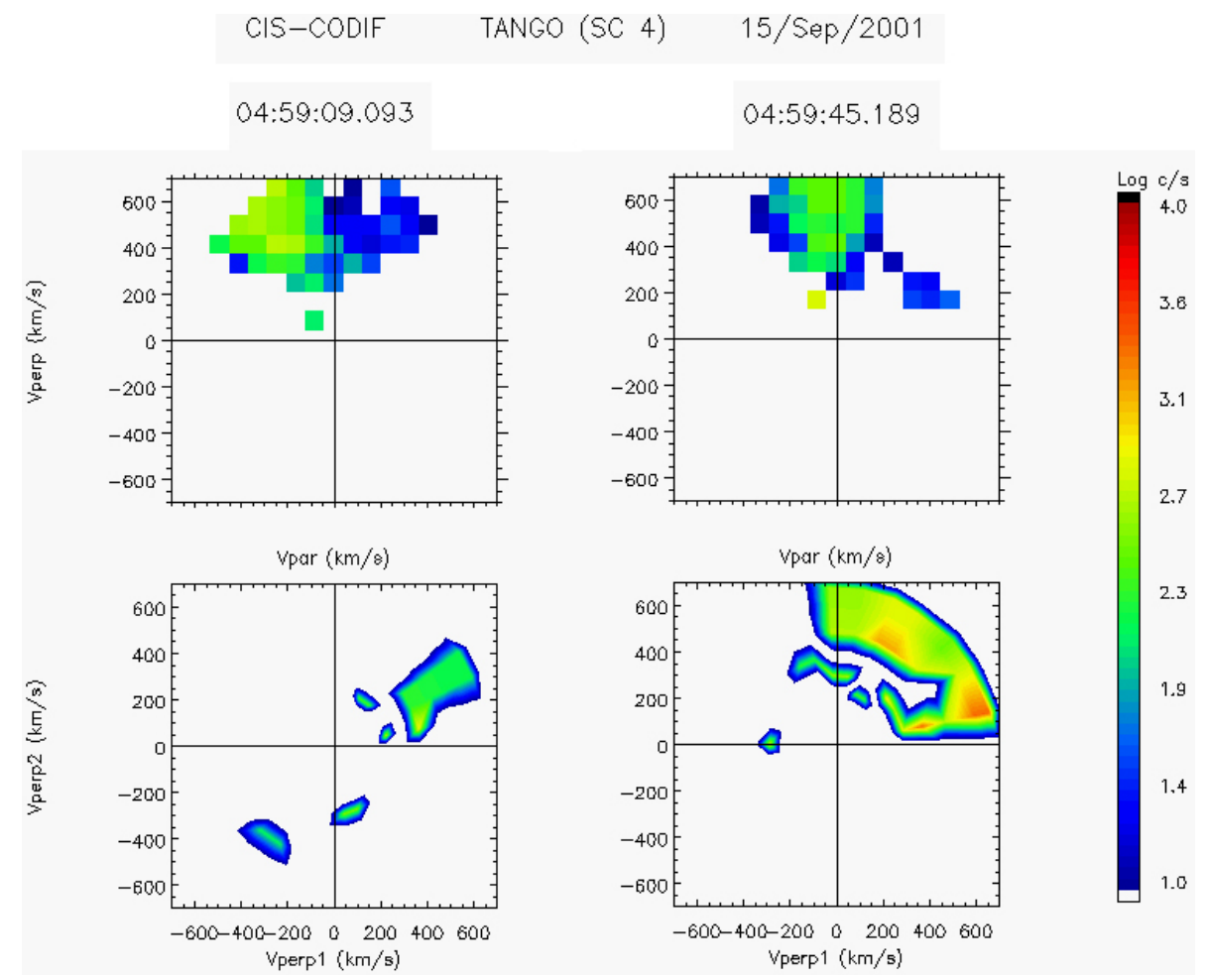

Fig. 9. Distribution functions of oxygen ions in the $V_{\|}-V_{\perp}$ coordinates from $\mathrm{C} 4$ in the bifurcated TCS.

\section{Discussion}

It is worth pointing out that although recently many observational investigations on TCS in the magnetotail were reported, few of them involved the particle kinetics. The observations presented here reveal the crucial role played by the ion kinetics in the dynamics of TCS. Particles in the region with a strong magnetic field gradient have totally different properties of motion in contrast to magnetic momentum adiabatic kinetics. The interaction of these nonadiabatic particles with the current sheet achieves eventually a selfconsistent TCS equilibrium. These equilibria manifest distinct behaviors from the Harris equilibrium, and in association with their intrinsic particle anisotropy and nongyrotropy. They are qualitatively in a good agreement with the conclusions given by these theoretical models considered into the so-called quasi-adiabatic sheet invariant (Sitnov et al., 2000, 2003, 2006).

The origin of TCS is unclear by now, although often referred to as the fast flow caused by the magnetic reconnection or substorm (Runov et al., 2003b; Asano et al., 2005). Here, the proton TCS was most likely due to field-aligned proton beams. But the source of these beams is also indistinct. During the present event, a substorm onset around 04:55 UT had been claimed (Voronkov et al., 2006). However, any obvious explosive plasma flow was absent in the beginning of the occurrence of the proton TCS. In panels $(\mathrm{g})$ and $(\mathrm{h})$ in Fig. 4, we notice that till 04:57:00 UT, no perceptible bulk proton flow was observed. A modest parallel flow was recorded once during the central line crossing at 04:57 UT. From 04:57:30 UT a modest perpendicular flow was observed. Likewise, in this period a modest oxygen ion flow was observed to be no more than $200 \mathrm{~km} / \mathrm{s}$. Similar measurements can also be found in Figs. 6 and 8. TCS observations without any fast plasma flow have also been reported in previous investigations (Sergeev et al., 2003; Asano et al., 2005).

The excitation mechanism of the TCS flapping motion is an argumentative issue, and seems not to match any occurrence that had been discussed so far in previous investigations. A heuristic approach on its ignition mechanism may be gained in the present event. In panel (b) in Fig. 4, from 04:54 UT to 05:02 UT, several small singular peaks of proton pressure was recorded, each corresponds to either the crossing of the central line or the stay at the outermost of TCS. Those peaks encountered across the central line are coming from density increases, and the corresponding small proton density peaks can be seen in panel (e). It indicates that density gradients exist in the center of TCS, in contrast to the case in thick sheets, in which the density gradient occurs only at the edge. Therefore, in the present event, the low hybrid drift instability is possible to develop from the observed density gradients. In the current sheet, the low hybrid drift instability was intensely investigated and also had been considered as the source of the current sheet flapping motion 


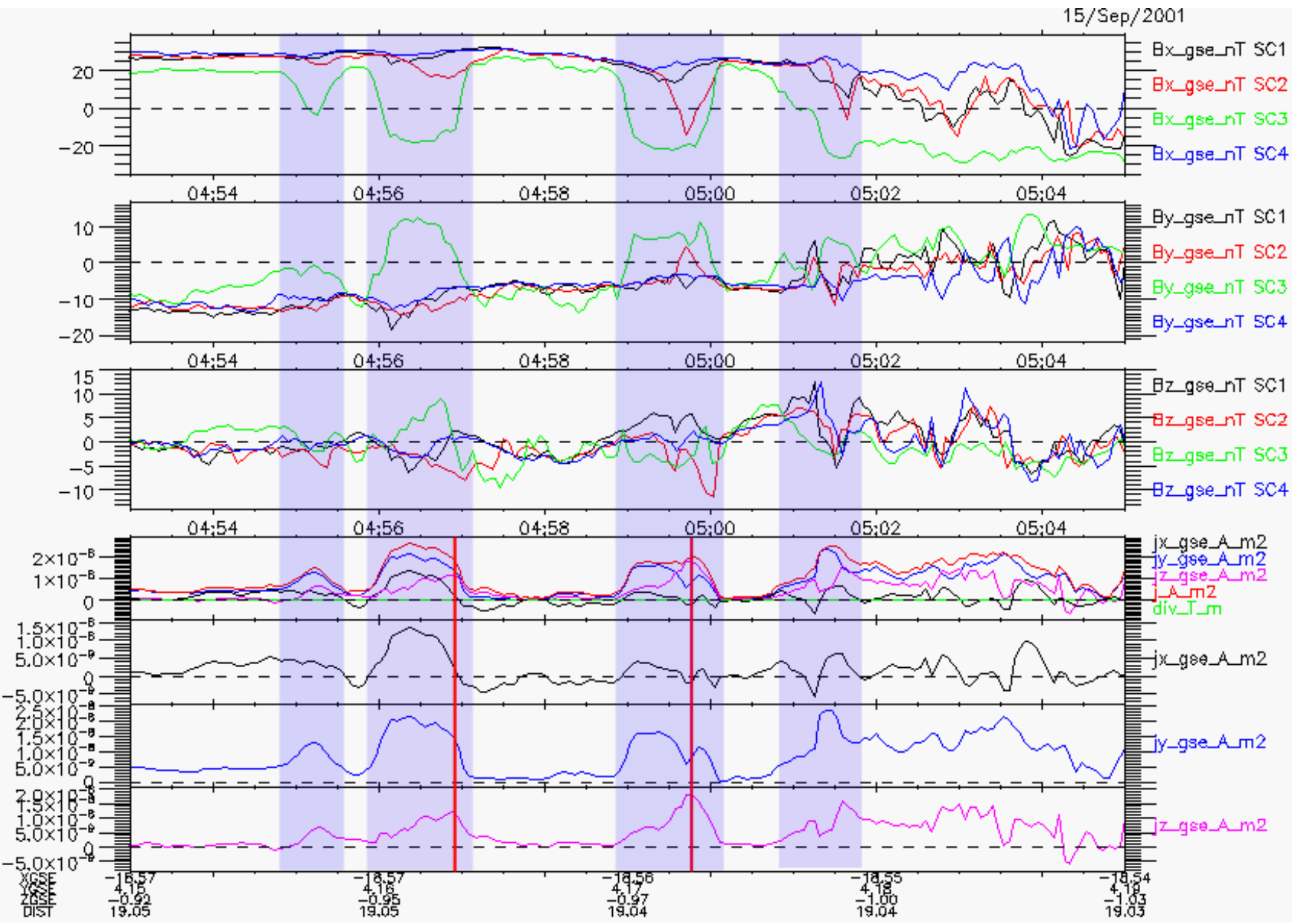

Fig. 10. Components of magnetic field for four satellites and the current density. Four large-amplitude magnetic variations due to flapping motion are labeled by the shadows.

(Daughton, 1999, 2002, 2003; Lapenta et al., 2002). Meanwhile, at 04:54:24 UT, before the ignition of TCS flapping, the first singular peak of proton pressure without any perceptible magnetic perturbation was recorded. It implies that the disturbance is a kinetic pressure pulse nearby the TCS at that time and will spread with the sound speed. When the fluctuation couples to the potentially excited low hybrid drift instability mentioned above, it might eventually evolve into a solitary wave modulated both on its amplitude and frequency via nonlinear interactions. Flapping motion is frequently not only associated with the TCS but also accompanied with the magnetic reconnection, both of them occurring in small scale. It also reflects, on the other hand, the meaningful role played by the low hybrid drift instability, which is easier to develop in TCSs than in thick sheets. Hence observations presented here also give insight into the generation mechanism of the TCS flapping motion.

\section{Conclusion}

During the Cluster spacecraft crossing of the magnetotail on 15 September 2001, both TCS embedding and bifurcation were recorded in one single event, due to the Cluster unique capabilities of spatial resolution on small scale. The intrinsic properties of nonadiabatic particle dynamics in the TCS are emphasized. It is indicated that the ion anisotropy and nongyrotropy are responsible for those new equilibrium features deviated from the conventional Harris model. An embedded proton TCS manifests a pressure anisotropy with $p_{\|}>p_{\perp}$, simultaneously associated with a density embedding, while a bifurcated oxygen ion TCS exhibits a pressure anisotropy mainly with $p_{\| \mid}<p_{\perp}$ and nongrotropy. Except at the edges $p_{\|}>p_{\perp}$ is the necessary marginal firehose stability condition. The local flapping motion of the TCS was observed, and some particular features such as the solitary wave-like behavior and the kink motion in the $y-z$ plane were revealed. A heuristic approach on its ignition mechanism is presented to support the notion that the TCS flapping 
motion is coming from a localized kinetic pressure pulse. The present investigation intimates the complexity of manifestations of magnetotail current sheet and displays the important role played by the ion kinetics in the self-consistent TCS equilibrium.

Acknowledgements. This work is supported by the National Natural Science Foundation of China (NSFC) under Grant No. 40574067, 40523006 and the National Basic Research Program of China 2006CB806305 and the French National Space Agency (CNES). C. L. Cai acknowledges the French Embassy in China for his government scholarship.

Topical Editor I. A. Daglis thanks two anonymous referees for their help in evaluating this paper.

\section{References}

Asano, Y., Nakamura, R., Baumjohann, W., Runov, A., Voros, Z., Volwerk, M., Zhang, T. L., Balogh, A., Klecker, B., and Rème, H.: How typical are atypical current sheets?, Geophys. Res. Lett., 32, L03108, doi:10.1029/2004GL021834, 2005.

Balogh, A., Carr, C. M., Acuña, M. H., et al.: The Cluster Magnetic Field Investigation: overview of in-flight performance and initial results, Ann. Geophys., 19, 1207-1217, 2001, http://www.ann-geophys.net/19/1207/2001/.

Birn, J., Schindler, K., and Hesse, M.: Thin electron current sheets and their relation to auroral potentials, J. Geophys. Res., 109, A02217, doi:10.1029/2003JA010303, 2004a.

Birn, J., Thomsen, M. F., and Hesse, M.: Acceleration of oxygen ions in the dynamic magnetotail, Ann. Geophys., 22, 1305-1315, 2004b, http://www.ann-geophys.net/22/1305/2004/.

Buchner, J. and Zelenyi, L. M.: Regular and chaotic charged particle motion in magnetotaillike field reversals: 1 . Basic theory, J. Geophys. Res., 94, 11 821-11 842, 1989.

Burkhart, G. R. and Chen, J.: Differential Memory in the Earth's magnetotail, J. Geophys. Res., 96, 14 033-14 049, 1991.

Camporeale, E. and Lapenta, G.: Model of bifurcated current sheets in the Earth's magnetotail: Equilibrium and stability, J. Geophys. Res., 110, A07206, doi:10.1029/2004JA010779, 2005.

Chen, J. and Palmadesso, P. J.: Chaos and Nonlinear Dynamics of Single-Particle Orbits in a Magnetotail-Like Magnetic Field, J. Geophys. Res., 91, 1499-1508, 1986.

Chen, J.: Nonlinear dynamics of charged particles in the magnetotail, J. Geophys. Res., 97, 15 011-15 050, 1992.

Cowley, S. W. H.: The effect of pressure anisotropy on the equilibrium structure of magnetic current sheets, Planet. Space Sci., 26, 1037-1061, 1978.

Daughton, W.: The unstable eigenmodes of a neutral sheet, Phys. Plasm., 6, 1329-1343, 1999.

Daughton, W.: Nonlinear dynamics of thin current sheets, Phys. Plasmas, 9, 3668-3678, 2002.

Daughton, W.: Electromagnetic properties of the lower-hybrid drift instability in a thin current sheet, Phys. Plasmas, 10, 3103-3119, 2003.

Delcourt, D. C., Malova, H. V., and Zelenyi, L. M.: Dynamics of charged particles in bifurcated current sheets: The k_1 regime, J. Geophys. Res., 109, A01222, doi:10.1029/2003JA010167, 2004.
Delcourt, D. C., Malova, H. V., and Zelenyi, L. M.: Quasiadiabaticity in bifurcated current sheets, Geophys. Res. Lett., 33, L06106, doi:10.1029/2005GL025463, 2006.

Eastwood, J. W.: Consistency of fields and particle motion in the "Speiser" model of the current sheet, Planet. Space Sci., 20, 1555-1568, 1972.

Fairfield, D. H.: On the average configuration of the geomagnetic tail, J. Geophys. Res., 84, 1950-1958, 1979.

Genot, V., Mottez, F., Fruit, G., Louarn, P., Sauvaud, J.-A., and Balogh, A.: Bifurcated current sheet: Model and Cluster observations, Planet Space Sci., 53, 229-235, 2005.

Harris, E. G.: On a plasma sheath separating regions of oppositely directed magnetic fields, Nuovo Cimento, 23, 115-121, 1962.

Hasegawa, A.: Plasma instabilities and nonlinear effect, SpringVerlag, New York, 1975.

Hoshino, M., Nishida, A., Mukai, T., Saito, Y., Yamamoto, T., and Kokubun, S.: Structure of plasma sheet in magnetotail: Doublepeaked electric current sheet, J. Geophys. Res., 101, 24775 24786, 1996.

Lapenta, G. and Brackbill, J. U.: Nonlinear evolution of the lower hybrid drift instability: Current sheet thinning and kinking, Phys. Plasmas, 9, 1544-1554, 2002.

Mottez, F.: Exact nonlinear analytic Vlasov-Maxwell tangential equilibria with arbitrary density and temperature profiles, Phys. Plasm., 10, 2501-2508, 2003.

Rème, H., Aoustin, C., Bosqued, J. M., et al.: First multispacecraft ion measurements in and near the Earth's magnetosphere with the identical Cluster ion spectrometry (CIS) experiment, Ann. Geophys., 19, 1303-1354, 2001, http://www.ann-geophys.net/19/1303/2001/.

Runov, A., Nakamura, R., Baumjohann, W., Zhang, T. L., Volwerk, M., and Eichelberger, H.-U.: Cluster observations of a bifurcated current sheet, Geophys. Res. Lett., 30(2), 1036, doi:10.1029/2002GL016136, 2003a.

Runov, A., Nakamura, R., Baumjohann, W., Treumann, R. A., et al.: Current sheet structure near magnetic X-line observed by Cluster, Geophys. Res. Lett., 30(11), 1579 , doi:10.1029/2002GL016730, 2003b.

Runov, A., Sergeev, V. A., Baumjohann, W., et al.: Electric current and magnetic field geometry in flapping magnetotail current sheets, Ann. Geophys., 23, 1391-1403, 2005, http://www.ann-geophys.net/23/1391/2005/.

Schindler, K. and Birn, J.: Models of two-dimensional embedded thin current sheets from Vlasov theory, J. Geophys. Res., 107(A8), 1193, doi:10.1029/2001JA000304, 2002.

Sergeev, V. A., Mitchell, D. G., Russell, C. T., and Williams, D. J.: Structure of the tail plasma/current sheet at $11 \mathrm{Re}$ and its changes in the course of a substorm, J. Geophys. Res., 98, 17 345-17365, 1993.

Sergeev, V., Runov, A., Baumjohann, W., Nakamura, R., Zhang, T. L., Volwerk, M., Balogh, A., Rème, H., Sauvaud, J. A., Andr'e, M., and Klecker, B.: Current sheet flapping motions and structure observed by Cluster, Geophys. Res. Lett., 30(6), 1327, doi:10.1029/2002GL016500, 2003.

Sergeev, V., Runov, A., Baumjohann, W., Nakamura, R., Zhang, T. L., Balogh, A., Louarn, P., Sauvaud, J.-A., and Rème, H.: Orientation and propagation of current sheet oscillations, Geophys. Res. Lett., 31, L05807, doi:10.1029/2003GL019346, 2004.

Sitnov, M. I., Zelenyi, L. M., Malova, H. V., and Sharma, A. 
S.: Thin current sheet embedded within a thicker plasma sheet: Self-consistent kinetic theory, J. Geophys. Res., 105, 13029 , doi:2001JA000287, 2000.

Sitnov, M. I., Guzdar, P. N., and Swisdak, M.: A model of the bifurcated current sheet, Geophys. Res. Lett., 30(13), 1712, doi:10.1029/2003GL017218, 2003.

Sitnov, M. I., Swisdak, M., Guzdar, P. N., and Runov, A.: Structure and dynamics of a new class of thin current sheets, J. Geophys. Res., 111, A08204, doi:10.1029/2005JA011517, 2006.

Sonnerup, B. U. O.: Adiabatic particle orbits in a magnetic null sheet, J. Geophys. Res., 76, 8211-8222, 1971.

Speiser, T. W.: Particle Trajectories in Model Current Sheets; 1. Analytical Solutions, J. Geophys. Res., 70, 4219-4226, 1965.

Thompson, S. M., Kivelson, M. G., Khurana, K. K., McPherron, R. L., Weygand, J. M., Balogh, A., Rème, H., and Kistler, L. M.: Dynamic Harris current sheet thickness from Cluster current density and plasma measurements, J. Geophys. Res., 110, A02212, doi:10.1029/2004JA010714, 2005.
Voronkov, I., Runov, A., Koustov, A., Kabin, K., Meurant, M., Donovan, E., Bryant, C., and Spanswick, E.: Features of magnetosphere-ionosphere coupling during breakups and substorm onsets inferred from multi-instrument alignment, Int. Conf. Substorms-8, 319, 2006.

Zelenyi, L., Delcourt, D., Malova, H., Sharma, A., Popov, V., and Bykov, A.: Forced current sheets in the Earth's magnetotail: Their role and evolution due to nonadiabatic particle scattering, Adv. Space. Res., 30, 1629-1638, 2002.

Zhang, T. L., Nakamura, R., Volwerk, M., Runov, A., Baumjohann, W., Eichelberger, H. U., Carr, C., Balogh, A., Sergeev, V., Shi, J. K., and Fornacon, K.-H.: Double Star/Cluster observation of neutral sheet oscillations on 5 August 2004, Ann. Geophys., 23, 2909-2914, 2005, http://www.ann-geophys.net/23/2909/2005/.

Zhu, Z. and Winglee, R. M.: Tearing instability, flux ropes, and the kinetic current sheet kink instability in the Earth's magnetotail: A threedimensional perspective from particle simulations, J. Geophys. Res., 101, 4885-4897, 1996. 